\title{
ESR Spectral Characterisation of Mn doped Zn (II) Trisethylenediamine Sulfidometalate Complexes
}

\author{
Beena K Vernekar
}

\begin{abstract}
Aqueous reaction of $\mathrm{Zn}(\mathrm{II})$ and $\mathrm{Mn}(\mathrm{II})$ salts with ammonium tetrasulfidometalate (NH4)2[MS4] $(M=M o, W)$ in the presence of ammonia or ethane 1,2-diamine (en) or propane 1,2-diamine (1,2-pn) results in the formation of $\left[M^{\prime}(L) n\right][M S 4]$ $\left(M^{\prime}=\mathrm{Zn}\right.$ or Mn; $L=N H 3$ or en or 1,2-pn). Using this strategy, Mn doped Zn complex of formula [Zn0.99Mn0.01(en)3][MS4], which exhibits characteristic hyperfine ESR spectra have been synthesized. The synthetic aspects, spectral and thermal characterization are described..
\end{abstract}

Keywords : Coordination chemistry, Tetrasulfidomolybdate, Zinc, Manganese, Hyperfine Spectrum

\section{INTRODUCTION}

The soluble sulphides of group VI metals Mo and W1 are unique as they exhibit a wide range of metal to sulfur stoichiometries, metal oxidation states, coordination and binding modes of sulfido ligands. Tetrasulfidometalates find use in therapy 2 and are employed as sulfur transfer reagents in organic syntheses 3 . The use of alkali metal and organic ammonium tetrasulfidometalates as useful probes for the advancement of $33 \mathrm{~S}$ solid state MAS NMR technique has added an entire new dimension4. The ammonium salts of [MoS4]2- or [WS4]2- routinely used as a starting material for the syntheses of a variety

of structurally diverse Mo-S and $\mathrm{W}-\mathrm{S}$ compounds, can function as pure inorganic ligands forming $\mathrm{S}$-bridged heterometallic compounds5. The ability of [MoS4]2- to coordinate transition metal centers like $\mathrm{Cu}+$ has led to its use as components in clusters and networks6.

Chemistry of formation of organic Tetrasulfidometalates which contain partially protonated organic amines as cations for charge balance has been exhaustively investigated by Srinivasan et al7. Recent addition to this structurally rich chemistry is the formation of two new hmt adducts (NH4)6[MS4]3·(hmt)48 [M = W (1), M = Mo (2) $]$. Ammonium tetrathiotungstate and bis(cetyltrimethylammonium) tetrathiotungstate are used as precursors for the soft synthesis of WS2 nanotubes9,10. The tetrahedral tetrasulfidometalates $[\mathrm{MS} 4] 2-(\mathrm{M}=\mathrm{Mo}, \mathrm{W})$ are well known for their use as starting materials for the preparation of a variety of metal sulfur compounds including hydrodesufurization (HDS) catalysts11. It has been reported

Revised Manuscript Received on December 31, 2019.

* Correspondence Author

Beena K Vernekar*, Department of Chemistry, Government College of Arts, Science \& Commerce, Khandola, Marcela - Goa 403107 India Email: beenavernekar@gmail.com that the HDS activity is enhanced when a bivalent $3 \mathrm{~d}$ metal like $\mathrm{Ni}(\mathrm{II})$ or $\mathrm{Co}(\mathrm{II})$ is added to Mo or $\mathrm{W}$ based sulphide catalysts and also that the active phase contains M'-Mo-Sunits $\left(\mathrm{M}^{\prime}=\mathrm{Ni}, \mathrm{Cu}\right) 12$. A material with the stoichiometry [Ni(en)3]MoS4 has been determined using a single crystal, prepared solvothermally13 and is characterized as an HDS precursor14. The highlight of this work, is the isolation of Mn doped [Zn(en3)][MS4] (M=Mo, W) which exhibits characteristic hyperfine ESR spectrum. It has been documented that the reaction of bivalent metal ions like $\mathrm{Zn} 2+$ with [MS4]2- leads to the formation of insoluble sulfides 15. In this work, we have investigated the reactions of the bivalent metals $\mathrm{Zn}$ (II) and $\mathrm{Mn}$ (II) with (NH4)2[MS4] (M=Mo, W) in the presence of ammonia and two organic diamines, ethane 1,2-diamine (en) or propane 1,2-diamine (1,2-pn) which results in the formation of [M'(L)n] [MS4]. The complexes are characterized on the basis of elemental analysis, IR, UV-vis, ESR, and thermal studies. This report indicates the emerging importance of [M'(L)n] [MS4] compounds which when decomposed at controlled temperature results into the formation of mixed metal sulphides which are potential materials and next generation catalysts.

\section{EXPERIMENTAL}

\section{A. Materials and methods}

The starting materials $\left(\mathrm{NH}_{4}\right)_{2}\left[\mathrm{MS}_{4}\right](\mathrm{M}=\mathrm{Mo}, \mathrm{W})$ were prepared by literature method ${ }^{9}$. Infrared spectra of the synthesized complexes were recorded (as $\mathrm{KBr}$ pellet) on a Shimadzu (IR Prestige-21) FTIR spectrometer at a resolution of $4 \mathrm{~cm}^{1-}$ referenced to polystyrene bands. UV-visible spectra were recorded in the region 200-800 nm using SHIMADZU UV-2450 UV-VIS spectrophotometer. ESR spectra were recorded at room temperature using a Varian E-109 spectrometer operating at X-band using DPPH as frequency calibrant. X-ray powder pattern were recorded on a PHILLIPS HOLLAND, PW-3710 diffractometer using $\mathrm{Cu}-\mathrm{K}_{\alpha}$ radiation. TG/DTA studies were performed on a SDT-2960 Thermal Analyser. Isothermal weight loss studies were performed in a temperature controlled furnace.

\section{B. Synthesis of Mn (II) doped $\left[\mathrm{Zn}\left(\mathrm{en}_{3}\right)\right]\left[\mathrm{MoS}_{4}\right](1)$}

The reaction of an aqueous solution containing a mixture of $\mathrm{ZnSO} 4 \cdot 7 \mathrm{H} 2 \mathrm{O}(284.66 \mathrm{mg}, 0.99 \mathrm{mmol})$ and $\mathrm{MnCl} 2 \cdot 4 \mathrm{H} 2 \mathrm{O}$ $(1.98 \mathrm{mg}, 0.01 \mathrm{mmol})$ in water ( 20 ml) with 
(NH4)2][MoS4] (260 mg, $1 \mathrm{mmol})$ or (NH3)2(WS4) (348 $\mathrm{mg}, 1 \mathrm{mmol})$ in water $(15 \mathrm{ml})$ and ethylenediamine $(\sim 1.0 \mathrm{ml})$, resulted in the immediate precipitation of bright red orange colored [Zn0.99Mn0.01(en)3][MoS4] (1) or the yellow W compound [Zn0.99Mn0.01(en)3][WS4] (1a). These products were isolated as earlier.

(1) IR data (vN-H)as 3292, (vN-H)s 3234, 2929, 2878, 1562, 1457, 1364, 1325, 1002, 630, 481, (vMo-S)472 cm-1. UV-Vis data (water) : $259 \mathrm{~nm}, 320 \mathrm{~nm}, 474 \mathrm{~nm}$. Anal. Calc. C,15.34; H, 5.15; N, 17.89; S, 27.30; Zn, 13.92\%. Found: C, 16; H, 4.78; N, 17.38; S, 28.00; Zn, 14.10\%.esr: g, 2.03; A ,86.(1a) IR data (vN-H)as 3295, (vN-H)s 3238, 2928, 2879, 1564, 1457, 1368, 1325, 1002, 634, (vW-S) 459 cm-1. UV-Vis data (water) : $282 \mathrm{~nm}, 395 \mathrm{~nm}$. Anal. Calc. C,12.92; H, 4.34; N, 15.07; S, 23.00; Zn, $11.60 \%$. Found: C, 13.30; H, 4.46; N, 14.78; S, 23.30; Zn, 11.55\%. esr: g , 2.01; A, 82

Synthesis of [Zn(L)n][MS4] (L = NH3, (en), (1,2-pn) and synthesis of $[\mathrm{Mn}(\mathrm{L}) \mathrm{n}][\mathrm{MS} 4](\mathrm{L}=\mathrm{NH} 3,(\mathrm{en}),(1,2-\mathrm{pn})$ is carried out as per the procedure given in supplementary data (S1).

\section{RESULTS AND DISCUSSION}

\section{A. Synthetic aspects}

It is well documented 15 that the aqueous reaction of bivalent 3d metal ions (M')2+ with [MS412- results in the precipitation of a mixture of X-ray amorphous $3 \mathrm{~d}$ metal sulfide and MS3 (see Scheme1). However, in the presence of a counter cation like $(\mathrm{PPh} 4)+$, bis(tetrasulfidometalato) complexes of the type [M'(MS4)2]2- $\left(\mathrm{M}^{\prime}=\mathrm{Fe}, \mathrm{Co}, \mathrm{Ni}, \mathrm{Zn}\right)$ charge balanced by the bulky cation are obtained17. Unlike other $3 \mathrm{~d}$ metal ions, reaction of aqueous solution of $\mathrm{Mn}(\mathrm{I1})$ with [MS4]2- neither immediately precipitates a mixture of amorphous metal sulfides nor forms any Mn-tetrasulfidometalate complexes in the presence of organic cations 18 .

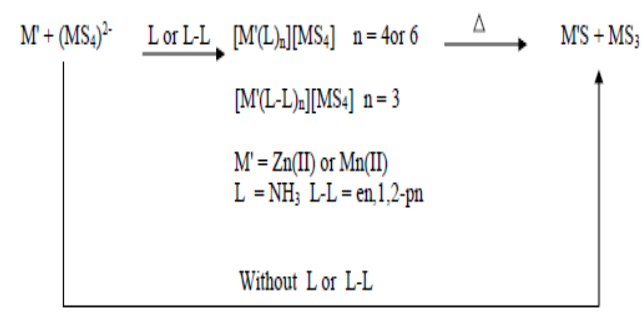

Scheme 1. Synthetic methodology

In view of the fact that [MS4]2- core is stable in an alkaline medium and does not retain its identity in acidic medium undergoing various reactions, the formation of insoluble sulfides can be attributed to the acidic nature of the aqueous solutions of $3 \mathrm{~d}$ metals. In order to prevent the formation of insoluble sulfides, the reactions of $\mathrm{Zn}(\mathrm{II})$ and $\mathrm{Mn}(\mathrm{II})$ with [MS4]2- have been investigated in this work in the presence of ammonia and two organic diamines (en) or (1,2-pn). The reaction of $\mathrm{Zn}(\mathrm{II})$ or $\mathrm{Mn}(\mathrm{II})$ with [MS4]2- in 1:1 mole ratio with excess ammonia results in the formation of the tetrammine zinc(II) compounds [Zn(NH3)4][MS4] ( $\mathrm{M}=$ $\mathrm{Mo}(2)$; W (2a)) while a hexamine manganese (II) is the charge balancing cation in [Mn(NH3)6][MS4] ( $\mathrm{M}=\mathrm{Mo}(5)$; $\mathrm{W}$ (5a). In contrast, for the reactions of $\mathrm{Zn}$ (II) or $\mathrm{Mn}$ (II) with [MS4]2- in 1:1 mole ratio in the presence of en or 1,2-pn resulted in the formation of tetrasulfidometalates charge balanced by the tris(diamine)metal(II) complex cation of the type $\left[\mathrm{M}^{\prime}(\mathrm{L}) 3\right]\left(\mathrm{M}^{\prime}=\mathrm{Zn}\right.$ or $\mathrm{Mn} ; \mathrm{L}=$ en or $\left.1,2-\mathrm{pn}\right)$. In view of the facile formation of [M'(en)3][MS4] $\left(\mathrm{M}^{\prime}=\mathrm{Zn}\right.$ or $\left.\mathrm{Mn}\right)$ compounds, reactions using a mixture of the bivalent metal ions $\mathrm{Zn}(\mathrm{II})$ and $\mathrm{Mn}$ (II) with [MS4]2- were investigated in the presence of en. These reactions afforded the synthesis of Mn doped $\mathrm{Zn}$ compounds (1) and (1a) and the presence of $\mathrm{Mn}(\mathrm{II})$ in these compounds was confirmed by a characteristic spot test for Mn(II) and characteristic ESR spectra. It is interesting to note that complexes of the type [M'(en)3][MS4] (M' = Ni or $\mathrm{Mn})$ have also been obtained under solvothermal conditions 19. It is to be noted that in the presence of aromatic diimmines like 2,2'-bipyridine (bpy), or 1,10-phenanthroline (phen), Mn(II) forms S-bridged bimetallic compounds containing the $\{\mathrm{Mn}(\mu-\mathrm{S}) 2 \mathrm{MS} 2\}$ unit18 where the [MS4]2functions as a bridging bidentate ligand. The presence of a free uncoordinated [MS4]2- unit in the compounds (1)-(7) and (1a)-(7a) can be inferred from the colour of the compounds (orange for Mo; yellow for $\mathrm{W}$ ) and characteristic uv-visible and infrared spectra. All our efforts to prepare S-bridged metal complexes by varying the reaction conditions and changing the stoichiometry of the bivalent metal and [MS4]2- reagents have not been fruitful and the compounds (1)-(7) and (1a)-(7a) are the only isolable products irrespective of the ratio of bivalent metal (excess) or [MS4]2(excess) employed. All the compounds prepared in this work, are analysed satisfactorily and the formulae of the compounds was arrived at based on elemental analytical data (Supplementary data Table S2). The compounds are insoluble in water and common organic solvents and dissolve in DMF or DMSO. The compounds are not stable in acid as evidenced by the formation of insoluble trisulfide MS3 with evolution of $\mathrm{H} 2 \mathrm{~S}$ on reaction with dilute $\mathrm{HCl}$. The acid decomposition reaction using excess $\mathrm{H}+$ thus provides a convenient method for the estimation of the bivalent metal content, which can be determined after filtering off the insoluble MS3.

\section{B. Spectral studies}

A combination of IR, UV-visible and ESR has been employed to characterize the synthesised complexes. The electronic spectra of the synthesized compounds (1)-(7) and (1a)-(7a) exhibit characteristic bands in the UV-Vis region (Supplementary data Table S4) and the observed signals can be assigned for the intraligand charge transfer transitions of the tetrahedral [MS4]2- chromophore17 (Supplementary Data Fig S5).

The infrared (IR) spectra of the synthesized compounds containing en or 1,2-pn exhibit several sharp bands in the mid-infrared region indicating the presence of the organic moieties ethane 1,2-diamine and propane 1,2-diamine (Supplementary data Table S3). 


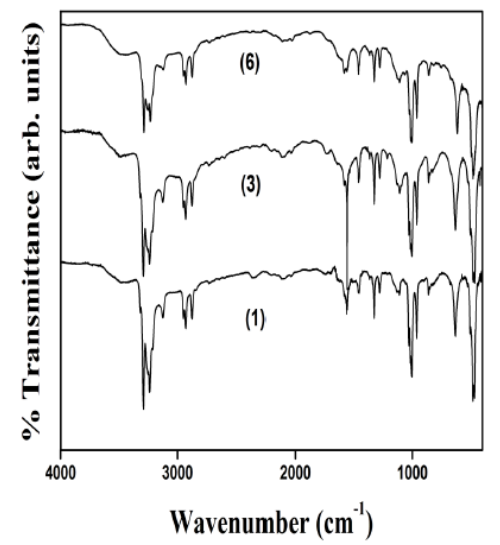

Fig.1 Comparative infrared spectra of $[\mathrm{Zn}(\mathrm{en})][\mathrm{MoS} 4](3),\left[\mathrm{Mn}\left(\mathrm{en}_{3}\right)\right][\mathrm{MoS} 4](5)$ and $\mathrm{Mn}$ doped

$\left[\mathrm{Zn}\left(\mathrm{en}_{\mathrm{n}}\right)\right][\mathrm{WS}$; $]$ (1)

All compounds exhibit an intense absorption peaks in the lower energy region assignable for the asymmetric stretching vM-S vibration in the free tetrasulfidometalate. This occurs at around $\sim 480 \mathrm{~cm} 1$ - and $\sim 460 \mathrm{~cm} 1$ - in [MoS4]2- and [WS4]2compounds respectively. The intense band in the region around $3100-3300 \mathrm{~cm} 1$ - can be attributed to $\mathrm{N}-\mathrm{H}$ symmetric and asymmetric stretching frequency of ammonia or organic diamine. The sharp peaks in the region 1300-1600 cm1-occur due to the symmetric and asymmetric $\mathrm{N}-\mathrm{H}$ bending vibrations. These vibrations are lowered in the region $1110-1250 \mathrm{~cm} 1$ in case of ammonia complexes. The intense band around 650$750 \mathrm{~cm} 1-$ in all the complexes is due to $\mathrm{N}-\mathrm{H}$ rocking vibrations. In the complexes (1), (1a), (3), (3a), (4), (4a), (6), (6a), (7), (7a), a strong band is observed around $1004 \mathrm{~cm}-1$ assignable to the $\mathrm{C}-\mathrm{N}$ stretching. The prominent bands in the region 2900-2800 $\mathrm{cm} 1-$ have been assigned due to asymmetric and symmetric $\mathrm{C}-\mathrm{H}$ stretching and bands around $1350-1460 \mathrm{~cm}-1$ are assigned to symmetric and assymmetic $\mathrm{CH} 2$ bending vibrations23. In complexes (4), (4a) and (7), (7a), symmetric and assymmetic $\mathrm{CH} 3$ bending vibrations are also observed in the region 1370-1460 cm-1(Supplementary data Table S3). The infrared spectrum of [ $\mathrm{Zn}(\mathrm{NH} 3) 4][\mathrm{MoS} 4]$ differs from that of the [Mn(NH3)6][MoS4] (Fig.1), which can be explained due to the differing coordination ( 4 for $\mathrm{Zn}$; 6 for $\mathrm{Mn}$ ) of the bivalent metal. The IR spectra of the [M'(en)3][MS4] (M = Mo, W; M' = Mn, Zn) complexes (Fig.1) are very similar indicating these compounds have similar structure and no change is observed by changing the bivalent metal. Similar pattern is observed for [M'(1,2-pn)3][MS4] (M = Mo, W; M' = Mn, Zn) complexes (Supplementary data Fig S7). The crystalline nature of these compounds was also inferred from their X-ray powder pattern (Supplementary data Fig. S18). It is to be noted that the infrared spectra of the $\left[\mathrm{M}^{\prime}(\mathrm{en}) 3\right][\mathrm{MoS} 4]\left(\mathrm{M}^{\prime}=\mathrm{Mn}, \mathrm{Zn}\right)$ compounds and Mn doped [Zn(en3)][MS4] (M=Mo, W) are almost identical in nature (Fig.1) but are different from the previously reported20 spectrum of (enH2)[MoS4], which can be attributed to the differing nature of the counter cations.

The $\mathrm{Zn}$ (II) compounds are diamagnetic while the $\mathrm{Mn}$ (II) compounds are paramagnetic and hence were studied by ESR spectroscopy. The esr spectrum of a paramagnetic substance is generally determined by operating at a fixed frequency and varying the field strength. In pure paramagnetic solids the spin-spin interaction between neighbouring magnetic dipoles causes the esr signal to be broadened. This broadening can be minimised by increasing the distance between the interacting dipoles. For this reason esr spectra are determined by using a paramagnetic material dissolved in large amount of diamagnetic solids. In view of this,paramagnetic Mn (II) has been doped in an isomorphous host complex [ $\mathrm{Zn}(\mathrm{en}) 3][\mathrm{MoS} 4]$. The ground state for $\mathrm{Mn}(\mathrm{II})$ in these complexes is found to be $6 \mathrm{~S} 5 / 2$. All the Mn compounds (5-7; $5 a-7 a)$ described in this work exhibit a single broad signal with a g value 2 (Supplementary data table S13, S14) in their ESR spectra at room temperature. The observed feature can be attributed to dipolar broadening as the spectra of pure polycrystalline (magnetically concentrated) samples were investigated. The ESR spectrum of [Mn(en)3][MoS4] (6) is shown in (Fig.2).

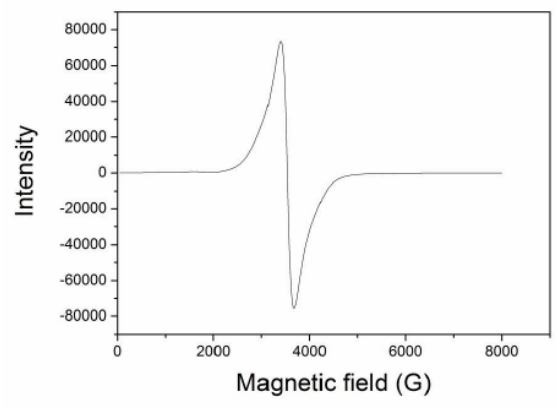

Fig. 2 First derivative ESR spectrum of polycrystalline $\left.[\mathrm{Mn}(\mathrm{en})]_{3}\right] \mathrm{MoS}_{4}(6)$

This observation is very similar to the reported ESR spectra of $\left[(\text { bpy })_{2} \mathrm{MnS}_{2} \mathrm{MS}_{2}\right.$ ] compounds ${ }^{18}$ which exhibit g values close to the free electron value. Unlike compounds (5-7) and (5a-7a), the Mn doped Zn compound (1,1a) exhibits a six line hyperfine spectrum (Fig.3). The observed A value of 86 gauss is in agreement with reported data ${ }^{18}$. The observation of a hyperfine ESR spectrum indicates that magnetic dilution has been achieved in solid state by doping $\mathrm{Mn}$ in the diamagnetic $\mathrm{Zn}$ (II) complex.

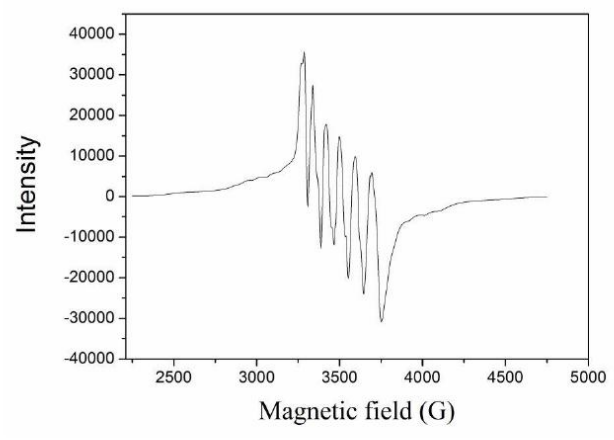

Fig. 3 First derivative ESR spectrum of Mn doped $\left[\mathrm{Zn}(\mathrm{en})_{3}\right] \mathrm{MoS}_{4}(\mathbf{1})$

\section{Thermal studies}

It is well documented that organic ammonium tetrasulfidometalates are useful precursors for sulphide materials (MS2) which can be prepared by thermal decomposition21. Hence the thermal decomposition studies of these complexes were investigated. The TG analysis of (2) showed two major mass loss steps between 150 oC -200 oC and $200 \mathrm{oC}-400 \mathrm{oC}$. 
The mass loss associated with this step is $18 \%$ in reasonable agreement with the $\%$ mass loss expected $19 \%$ indicative of the loss of four ammonia molecules (Supplementary data Fig. S15). The probable solid after, ligand loss would be M'MS4, ie MS.MoS3. MoS3 has been reported to decompose to MoS2 and S at 310 oC 24 and the boiling point of $\mathrm{S}$ is $440 \mathrm{oC}$ 25 . In view of this the second mass loss step in (2) is attributed to the conversion of MoS3 to MoS2 and S19.The W analogue undergoes the similar trend.TG pattern for compound (5) differs from (2) and could be attributed to its hexacoordinate nature, \% mass loss in the first step is 27.9 in agreement with the $\%$ mass loss of 26.8 for six molecules of ammonia which are lost in the region $100 \mathrm{oC}-400 \mathrm{oC}$. This observation is consistent with the IR pattern of decomposed complexes (2), (2a), (5), (5a) at 200oC (supplementary data Fig. S8). Based on the featureless IR spectrum above $400 \mathrm{oC}$ for these complexes the decomposed products can be formulated as [M'S.MS3].

It has been observed in the complexes (3), (3a), (4), (4a), (6), (6a), (7), (7a) that the coordinated diamine is lost in two steps. In the first step the mass loss approximately corresponds to two diamines in the range $200 \mathrm{oC}-400 \mathrm{oC}$ giving rise to phase [M'enMoS4] which exhibits an infrared spectrum (Supplementary Data Fig. S16, Fig.17) similar to that of the catena-M-ethylenediamine complex $[\mathrm{M}(\mathrm{en}) \mathrm{Cl} 2] 22$. Subsequently followed by the loss of third coordinated diamine above $400 \mathrm{oC}$. This ultimately results in the formation of mixed metal sulphides in the second step.This observation is also supported by the presence of characteristic $\mathrm{N}-\mathrm{H}$ and $\mathrm{C}-\mathrm{H}$ vibrations in the IR spectra of the decomposed products at $200 \mathrm{oC}$ (supplementary data fig S9, fig.S10) and $400 \mathrm{oC}$ (supplementary data Fig. S11, Fig.S12). This study is consistent with the mass loss data (table 1).

\begin{tabular}{|c|c|c|c|c|c|}
\hline compound & Temp range ${ }^{\circ} \mathrm{C}$ & $\begin{array}{l}\text { Experimental } \\
\text { weight loss } \\
\%\end{array}$ & $\begin{array}{l}\text { Probable } \\
\text { products }\end{array}$ & $\begin{array}{l}\text { Calculated } \\
\text { weight } \\
\text { loss } \%\end{array}$ & DTA peak \\
\hline$\left[\mathrm{Zn}\left(\mathrm{NH}_{3}\right)_{4}\right] \mathrm{MoS}_{4}$ & $150-200$ & 18.00 & $\mathrm{ZnSMoS}_{3}$ & 19.02 & 145 endo \\
\hline$\left.\left[\mathrm{Mn}\left(\mathrm{NH}_{3}\right)\right)_{3}\right] \mathrm{MoS}_{4}$ & $100-400$ & 27.86 & $\mathrm{MnSMoS}_{3}$ & 26.77 & 100 exo \\
\hline \multirow[t]{2}{*}[\mathrm{Zn}(\mathrm{en})]{$\mathrm{MoS}_{4}$} & $150-370$ & 25.97 & $\mathrm{Zn}(\mathrm{en}) \mathrm{MoS}_{4}$ & 25.56 & 200 endo \\
\hline & $450-510$ & 36.00 & $\mathrm{ZnSMoS}_{3}$ & 38.34 & 485 endo \\
\hline \multirow[t]{3}{*}[\mathrm{Zn}(\mathrm{en})_{3}]{$\mathrm{WS} 4$} & 200-400 & 20.80 & $\mathrm{Zn}(\mathrm{en}) \mathrm{WS}_{4}$ & 21.52 & 200 endo \\
\hline & $400-600$ & & $\mathrm{ZnSMoS}_{3}$ & 32.30 & 475 endo \\
\hline & $200-400$ & 32.58 & $\mathrm{Mn}(1,2 \mathrm{pn}) \mathrm{MoS}_{4}$ & 30.00 & 170 exo \\
\hline \multirow[t]{2}{*}[\mathrm{Mn}(1,2-\mathrm{pn})]{$\mathrm{MoS}_{4}$} & $200-600$ & & $\mathrm{MnSMoS}_{3}$ & 44.31 & 600 exo \\
\hline & $200-470$ & 26.00 & $\mathrm{Mn}(1,2 \mathrm{pn}) \mathrm{WS}_{4}$ & 25,13 & 170 exo \\
\hline$\left[\mathrm{Mn}(1,2-\mathrm{pn})_{3}\right] \mathrm{WS}_{4}$ & $200-800$ & 36.00 & $\mathrm{MnSMoS}_{3}$ & 38.00 & 480 exo \\
\hline
\end{tabular}

\section{Conclusions}

The present work describes a methodology for the facile synthesis of complexes obtained by reaction of cationic salts charge balanced by (MS4)-2 anions of the formula [M'(L)n][MS4] $\left(\mathrm{M}^{\prime}=\mathrm{Zn}(\mathrm{II})\right.$ and $\mathrm{Mn}(\mathrm{II})$; L= NH3 or en or 1,2-pn; $\mathrm{M}=\mathrm{Mo}$ or $\mathrm{W})$. The complexes thus prepared have been characterized by analytical data, thermal, uv-visible, ESR and infrared spectra.The Mn(II) compounds exhibit broad ESR signals while the magnetically diluted (Mn-doped) $\mathrm{Zn}$ (II) compound exhibits a hyperfine spectrum. The thermal studies of these complexes provides easy route to their characterization as the ammonia and diammines are lost at particular temperature and the decomposition products are the mixed metal sulphides which are the potential materials and next generation catalysts.

\section{Supplementary Data}

Supplementary Data associated with this article are available in the electronic form at http://www.niscair.res.in/jinfo/ijca/IJCA 51A(XX) YYYY-YYYY_Suppl Data.pdf

\section{ACKNOWLEDGEMENTS}

Acknowledgements: BKV acknowledges deep sense of gratitude to Prof. B. R. Srinivasan, Department of Chemistry, Goa University, for introducing her to the chemistry of Tetra Sulfidometalates. She thanks Dr Savita Khandolkar, Dept of Chemistry, Goa University, Goa for all the help rendered.

\section{REFERENCES}

1. T. B. Rauchfuss, Inorg. Chem. 43(2004) 14; T. Shibahara, Coord Chem. Rev. (1993) 73; D. Couccovanis, Adv. Inorg. Chem. 1(1998) 45.

2. a) S. Bag, A. F. Gaudette, M. E. Bussell \& M. G. Kanatzidis, Nature Chemistry, 1 (2009) 217; b) M. Polyakcov, S. Indris, S. Schwaborn, A. Mazeika, M. Poisot, L. kienle, W. Bensch, M. Muhler, W.Grünert, J Catal, 260 (2008) 236

3. a) F.Askari, D.Innis, R. B. Dick, G. Hou, J. Marrero, J. Greenson \& G. J. Brewer, Trans Res, 155 (2010) 123; b) G. J. Brewer, Drug discovery Today, 10 (2005)1103.

4. K. R. Prabhu, N. Devan \& S. Chandrasekaran, Synlett, (2002) 1762.

5. E. Diemann , A. Müller, Coord. Chem. Rev.10 (1973) 79; b) T. Shibhahara, Coord. Chem. Rev.123 (1993) 73; c) T.B. Rauchfuss, Inorg. Chem. 43 (2004) 14.

6. a)Y.Y. Niu, T. N. Chen, S. X. Liu, Y. L. Song, Y. X. Wang, Z. L. Xiun. X.Q. Xue, J. Chem Soc, DaltonTrans.(2002)1980; b) W. H. Zhang, J. P. Lang, Y. Zhang, B.F. Abrahams, Cryst. Growth Des, 8 (2008) 399.

7. a)H. J. Jakobsen, H. Bildsoe, J. Skibsted, M. R. Hansen, M. Brorson, B. R. Srinivasan W. Bensch, Inorg Chem, 48 (2009) 1787; b) H. J. Jakobsen, H. Bildsoe, J. Skibsted, M. Brorson, B.R. Srinivasan, C. Näther, W. Bensch, Phys Chem Chem Phys, 11(2009) 6981; c) B. R. Srinivasan, A. R. Naik, M. Poisot, C. Näther, W. Bensch, Polyhedron 28 ( 2009), 1379; d) B. R. Srinivasan, A. R. Naik, S. N. Dhuri, C. Näther, W.Bensch, Polyhedron. 28 (2009) 3715; e) B. R. Srinivasan, S. N.Dhuri, C. Näther, W. Bensch, Trans. Met. Chem. (32) 2007,64; f) B. R. Srinivasan, S. N. Dhuri, A. R. Naik, C. Näther, W. Bensch, Polyhedron 27 ( 2008 ) 25; g) B. R. Srinivasan, S.V Girkar, C. Näther, W. Bensch J. of Coordination Chemistry, 63 (2010) 931.

8. B.R. Srinivasan, S.N. Dhuri, A.R. Naik, C. Näther, W. Bensch, Z.Anorg. Allg. Chem. 639 (2013) 512.

9. a) X. M. Nath, A. Govind Ray, C.N. Rao, Adv. Mater, 15 (2001)283, b) J. Chen, S. L. Li, F. Gao, Z. L.Tao, Chem Mater, 15 (2003)1012.

10. Y. D. Li, X. L. Li, R. R. He, J. Zhu, J. Am. Chem. Soc. 124 (2002) 1411.

11. a) M. Poisot, C. Näther, W. Bensch, Z. Naturforsch, 62b (2007) 209; b) B. R. Srinivasan, A.R. Naik, S. N. Dhuri, C. Näther, W. Bensch, Polyhedron, 28 (2009) 3715; c) B. R. Srinivasan, S. N. Dhuri, A. R. Naik, C. Näther, W. Bensch, Polyhedron, 27 (2008) 25.

12. T. C. Ho, W. H. Pan, A. J. Jacobson, R. R. Chianelli, Appl. Catal. A 421(2012) 38 .

13. J. Ellemeier, C. Näthar, W. Bensch, Acta. crystallogr. C55 (1999)501.

14. F. Brezina, P. Kopel, Z. Sinderal, R. Pastorek, Transition Met. Chem. 20 (1995) 56, T.C. Ho, Catal. Today 130( 2008) 206.

15. G. M. Clark, W. P. Doyle, J. Inorg. Nucl. Chem. 28 (1966) 381

16. J. W. McDonald, G. D. Friesen, L. D. Rosenhein, W. E. Newton, Inorg. Chim. Acta. 72 (1983) 205.

17. A. Müller, E. Diemann, R. Jostes, H. Bögge, Angew. Chem. Int. Ed. Engl. 20 (1981) 934. 
18. B. R. Srinivasan, S. Sarkar, Inorg. Chem. 29 (1990) 3898.

19. H. Tian, H. A. Iliff, L. J. Moore, C. M. Oertel, Cryst. Growth Des.10 (2010) 669.

20. B. R. Srinivasan, B. K. Vernekar, K. Nagarajan, Indian J. Chem. 40A (2001)563.

21. a) M. Poisot, W. Bensch, Fuentus, G. Alonso, Thermochim. Acta. 444 (2006) 35; b) M. Poisot, W. Bensch, Thermochim. Acta. 453 (2007) 42.

22. T. Iwamoto, D. F. Shriver, Inorg. Chem.10 (1971) 2428

23. K.Nakamoto, Infrared and Raman Spectra of Inorganic and Coordination Compounds Part A: Theory and Applications in Inorganic Chemistry, sixth ed. John Wiley New Jersey, 2009, p. 204.

24. T.Weber, J. C. Muijsers, J. W. Niemantsverdriet , J. Phys. Chem. 99 (1995) 9194

25. N. N.Greenwood, A. Earnshaw, Chemistry of the elements, $2^{\text {nd }}$ edition Elsvier Butterworth Heinemann Boston, 997. 